\title{
A Comparative Study on Efficacy of 30\% Trichloroacetic Acid versus 35\% Glycolic Acid Peel in the Treatment of Melasma
}

\author{
Begum $\mathrm{ST}^{1}$, Khan $\mathrm{MAL}^{2}$, Hasan $\mathrm{MS}^{3}$, Khan $\mathrm{MSI}^{4}$, Karim $\mathrm{ATMR}^{5}$
}

\begin{abstract}
Introduction: Melasma is a common hyperpigmented disorder of skin which has severe impact on the quality of life. Many modalities of treatment are available to treat melasma. Among them chemical peeling is one of the most popular and widely used method of treatment for melasma.
\end{abstract}

Objective: The present study was aimed to assess the efficacy of $30 \%$ trichloroacetic acid versus $35 \%$ glycolic acid peel in the treatment of melasma.

Materials and Methods: The prospective randomized study included 50 patients of melasma of both sexes, in 20-55 years age group. Patients were randomly divided into two equal groups $A$ and $B$. Group A was treated with trichloroacetic acid (TCA $30 \%$ ) peel and group B with glycolic acid (GA $35 \%$ ) peel. Five peels were done serially at intervals of 21 days. Patients were followed up every 3 weekly for 6 weeks after the last peel. The disease severity was monitored with digital photography and melasma area and severity index (MASI) score which were calculated at baseline, 6 weeks, 12 weeks and 18 weeks. A visual analog scale (VAS) was calculated at baseline, 12 weeks and 18 weeks.

Results: Glycolic acid showed better response compared to trichloroacetic acid at the end of five peels, but this difference was not statistically significant $(p>0.05)$. Chemical peeling with trichloroacetic acid produced significantly more erythema, burning sensation $(p<0.05)$ and higher incidence of post peel inflammatory hyperpigmentation $(p<0.05)$ compared to glycolic acid.

\section{Introduction}

Melasma is a common acquired symmetric hypermelanosis characterized by irregular light to grey brown macules involving sun exposed areas ${ }^{1}$. The pathogenesis of melasma is not fully understood but pregnancy, estrogen ingestion, ultraviolet (UV) light exposure, and family history are well recognized association ${ }^{2}$. Topical hydroquinone is the most common treatment of melasma. Other treatment modalities include retinoic acid (tretinoin), kojic acid, azelaic acid and combination of hydroquinone, tretinoin and corticosteroids ${ }^{3}$. Now a day's chemical peel, laser treatment and intense pulsed light therapy are widely used popular method of treatment for melasma. Among them, chemical peeling provides more rapid response in treating melasma. Chemical peels are used to create injury at a specific skin depth with the goal of stimulating new skin growth and improving surface texture ${ }^{4}$.Chemical peels are classified by the depth of action into superficial, medium, and deep peels. Specific peeling agents should be selected based on the disorder to be treated and determined by the histological level or severity of skin pathology to maximize the outcome. Today plethora of peeling agents is available ${ }^{6}$. The most commonly used chemical peeling agents include Phenol, Trichloroaceticacid (TCA), Alpha Hydroxyacids(AHAs) eg. Glycolic acid (GA) and Beta Hydroxyacids eg. Salicylic acid. Although both TCA and $\mathrm{GA}$ are being used in various centers, there have been very few studies comparing these two agents. We undertook this study to compare the therapeutic response of $35 \%$ glycolic acid (GA $35 \%$ ) versus $30 \%$ trichloroacetic acid (TCA 30\%) on melasma.

1. Maj Syeda Tania Begum, MBBS, DDV, FCPS, Classified Specialist in Dermatology and Venereology, CMH, Dhaka

2. Brig Gen Md Abdul Latif Khan, MBBS, DDV, FCPS (Dermatology \& Venereology), Adviser and Head, Department of Dermatology and Venereology, CMH, Dhaka 3. Col Md Sayeed Hasan, MBBS, DDV, FCPS, Senior Classified Specialist and Associate Professor, Dermatology and Venereology, Armed Forces Medical College, Dhaka 4. Lt Col Md Shirajul Islam Khan, MBBS, DDV, MCPS, FCPS, Classified Spl in Dermatology \& Venereology, CMH, Dhaka 5. Lt Col ATM Rezaul Karim, MBBS, DDV, MCPS, FCPS, Classified Spl in Dermatology, CMH, Comilla. 


\section{Materials and Methods}

This was a prospective randomized study of 18 weeks duration conducted during the period from May 2015 to October 2015. Fifty patients of melasma attending the dermatology outpatient department at $\mathrm{CMH}$ Dhaka were selected into the study and were randomly allotted to Group-A and Group-B. All the patients of both sexes and between the ages of 20-55 years were included. Pregnant or lactating females, patients with a known hypersensitivity to chemical peel, patients applying topical retinoids, females taking oral contraceptive pill and patients with active infection were excluded. An informed consent was taken from all the patients. Detailed history regarding the duration and extent of the disease, family history, past treatment and aggravating or initiating factors were recorded. Group A comprised of 25 patients who received peeling with trichloroacetic acid (TCA-30\%) and Group B comprised of 25 patients who received glycolic acid peel (GA-35\%). In Group A, Peeling agent was kept until the appearance of frosting and in Group B for three minutes. Both the peeling agents were then neutralized with cold water. Before applying the chemical agent the face was washed with soap and water and then degreasing was done with commercially available alcohol pad. No pre peel treatment was done. A post auricular test peel was performed and left for 15-20 minutes to find any hypersensitivity in all patients. After peeling all patients were advised strict sun protection and liberal use of emollients and sunscreen with SPF 50. Clinical examination including Melasma Area and Severity Index (MASI) scores of all the patients were noted. MASI score was calculated ranking the severity of melasma in terms of its Darkness (D), Homogeneity $(\mathrm{H})$ of appearance and the percentage Area of the face affected $(A)$ and then using the following formula:

MASI $=0.3(\mathrm{DF}+\mathrm{HF}) \mathrm{AF}+0.3(\mathrm{D} M R+\mathrm{H} M R) A$ $M R+0.3(D M L+H M L) A M L+0.1(D C+H C) A C$.

Darkness was ranked from 0 to 4 , Homogeneity from 0 to 4 and Area from 0 to 6 . MASI score was calculated at baseline, 6 weeks, 12 weeks and 18 weeks. The lesions were photographed with and without flash with a standard 5 mega pixel digital camera at $30 \mathrm{~cm}$ distance and approximately $2 \mathrm{MB}$ resolution.
In addition, Visual Analog Scale (VAS) ranging from 0 to +10 was assessed by comparing follow-up images to the baseline photograph pretreatment. VAS were assessed by the dermatologist colleague who was blinded for the study. On the VAS, 0 represents no pigmentation and 10 represents maximum pigmentation. VAS measurements of photographic documentation were available at time points 0 (baseline),12 weeks and 18 weeks. Five peels were done serially at intervals of 21 days for every patient. Patients were followed every 3 weekly for 6 weeks after the last peel in both the groups. The outcome of treatment was calculated by comparing the mean MASI scores in the two groups at 6 weeks, at 12 weeks and 18 weeks. In addition, efficacies of each treatment agents were evaluated by comparing the reduction in mean MASI at 12 weeks from baseline and comparing the mean VAS at 12 weeks. All the data collected were then analyzed by standard data analysis software's.

\section{Results}

There were 50 patients included in the study with 47 females and only 3 males, in the age range 20 years and 55 years with a mean of $34.3 \pm 5.9$ years. Duration of melasma ranged between 1 and 8 years with a mean $3.2 \pm 2.3$ years. There was no significant precipitating factor observed in relation to occurrence of melasma. Most cases (84\%) were of epidermal type and one-fourth (16\%) of the cases were of mixed type of melasma. The most common pattern was malar $(78 \%)$ followed by centrofacial pattern (24\%) and mandibular (10\%). Figure-1 shows the MASI scores at baseline 6,12 and 18 weeks. Response to treatment in MASI scoring after 12 weeks was $78 \%$ reduction (from 26.5 to 5.9 ) in GA group and $79 \%$ reduction (from 28.9 to 6 ) in TCA group.

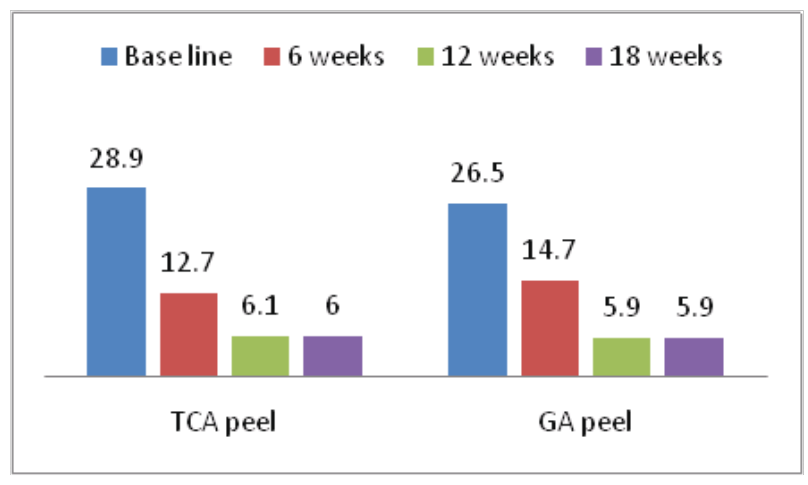

Fig-1: MASI score objective assessment in both groups. 
There was no significant difference in reduction of MASI scores at the end of five peels after 12 weeks between both the groups $(P>0.05)$. However, TCA peel showed an initial rapid response compared to GA but results were statistically insignificant. In the TCA group, the patients reported a quicker improvement but at the end of peeling sessions, both the groups showed equally efficacious response. Based on the extent of improvement, the patients were arbitrarily classified into one of the following categories: very good if there was $>75 \%$ reduction from baseline VAS; good if VAS reduced by $50-74 \%$; moderate if VAS reduced by $25-49 \%$ and mild if $<25 \%$ reduction in VAS was achieved. Table-1 reveals good and very good response was $76 \%$ in GA group and was $68 \%$ in TCA group (statistically insignificant).

Improvements shown according to VAS in both groups at the end of peeing sessions

\begin{tabular}{c|c|c|}
\hline ntage Improvement & TCA & GA \\
\hline good $(>75 \%)$ & 4 & 7 \\
\hline$(50-75 \%)$ & 13 & 12 \\
\hline rate $(25-50 \%)$ & 3 & 3 \\
\hline$<25 \%)$ & 5 & 3 \\
\hline
\end{tabular}

During the study, the frequencies of serious side effects were very low. Table-Il shows mild burning sensation in $92 \%$ of patients in GA group and $8 \%$ experience moderate burning but there were moderate to severe burning in $92 \%$ of patients in the TCA group and mild burning in $8 \%$ of the patients. Post peel crackening and hyperpigmentation were reported in $48 \%$ of cases in TCA group but none of the patients in the GA group (Table-II).

Complication during peeling and follow up period

\begin{tabular}{c|c|c|c}
\hline cation & TCA & GA & P value \\
\hline rning & $2(8 \%)$ & $23(92 \%)$ & $<0.5$ \\
\hline ate burning & $6(24 \%)$ & $2(8 \%)$ & $<0.5$ \\
\hline burning & $17(68 \%)$ & 0 & $>0.5$ \\
\hline $\begin{array}{l}\text { el inflammatory } \\
\text { gmentation }\end{array}$ & $12(48 \%)$ & 0 & $>0.5$ \\
\hline
\end{tabular}

There was significant improvement in the texture and glow of skin appreciated by $76 \%$ of the patients in GA group, which was not appreciated in TCA group. No relapse was seen within follow up period.

\section{Discussion}

Melasma is more common in women of childbearing age, although men also suffer from the condition and account for $10 \%$ of the cases ${ }^{8,9}$. Melasma affects all races, but is observed more frequently among individuals with skin type IV-VI, especially in woman, who live in areas of intense ultraviolet radiation ${ }^{10}$. There are three clinical patterns-centrofacial, malar, and mandibular-depending upon the area of localization. Histologically, melasma is divided into three types: epidermal, dermal, and mixed $^{11}$. Chemical peeling aims at production of controlled chemical burn of epidermis and/or dermis, resulting in resurfacing of epidermis and remodeling of collagen and elastic fibre with deposition of glycosaminoglycans in dermis. Both the agents used in this study, TCA $30 \%$ and GA $35 \%$, are superficial peels ${ }^{11}$. In this study, the average age of patients and duration of the disease is comparable to studies from India ${ }^{12}$. The types of melasma in our patients are also similar to the study from India probably due to same skin type ${ }^{14}$. In this study and most other studies the commonest pattern was malar followed by centrofacial ${ }^{12,13}$. In another Indian study carried out by Kalla et al GA and TCA showed comparable results on subjective scores given by patients ${ }^{14}$. They had not used any scientific scoring system like MASI for comparison. In this study both the chemical agents used for chemical peel shows comparable results depending on MASI and VAS score and none has statistically significant better result than other (Figure-1). Kalla et al observed a more rapid response to TCA than GA in their study ${ }^{14}$. As with our study the local irritant effects and post peel crackening were more with TCA than with GA. Relapse and hyperpigmentation were much less in this study. The rate of post peel inflammatory hyperpigmentation was higher in the TCA group $(48 \%)$ than GA group and statistically significant (Table-2). TCA group of patients also complain more burning sensation than GA group. In a recent study from Pakistan, The mean score of response calculated for both the groups revealed better overall clinical response in TCA group than in GA group but this difference was statistically insignificant $(P>0.05)$ comparable to our study ${ }^{15}$. Limitation of our study included the observer bias in the subjective scoring. To eliminate this, MASI scoring was done by a single-blinded independent 
person. Post inflammatory hyperpigmentation has been reported to be the most common side effect with GA facial peels ${ }^{12}$ but in this study the frequency of all side effects was much lower as compared to that of other studies ${ }^{11,14}$. This may have been due to the specific time of application, method of application and strict adherence to sun protection. No patient developed herpes, vesiculation or post peel keloid as was reported in other studies. Regular use of sunscreen helped in maintaining the result of the peels on follow up. Post peel crackening effect that occurred only with TCA and not with GA makes it beneficial for patients to continue outdoor activities and office work in GA group.

\section{Conclusion}

$35 \%$ GA and $30 \%$ TCA concentrations of facial peel are effective treatment for melasma with significant improvement and without any major side effects. The beneficial results achieved can be maintained with topical application of sunscreen SPF-50. Regular use of sunscreens prevents the chances of recurrence of melasma. GA peel is associated with fewer side effects than TCA and has the added advantage of facial rejuvenation and does not hamper patient's daily routine.

\section{References}

1. Grimes PE. Melasma: Etiologic and Therapeutic considerations. Arch Dermatol 1995; 131:1453-7.

2. Thappa DM. Melasma (chloasma): A review with current treatment options. Indian J Dermatol 2004; 49:165-76

3. Drs William D, James, Timothy G, Berger and Dirk M. Elston. Andrews'. Disturbances of pigmentations: Diseases of the Skin: Clinical Dermatology. Saunders Elsviere 2006; 36:858.

4. Ghersetich P, Teofol LM, Gantcheva M. Chemical peeling: How, when, why? J Eur Acad Dermatol Venererol 1997; 8:1-11.
5. Wiest L. Chemical peels in aesthetic dermatology. Hautarzt 2004; 55:611-20.

6. Konda S, Geria AN, Halder RM. New Horizons in Treating Disorders of Hyperpigmentation in Skin of Color. Seminars in Cutaneous Medicine and Surgery 2012; 31(2):133.

7. Gilchrest BA, Fitzpatrick TB, Anderson RR, et al. Localization of melanin pigmentation in the skin with Wood's lamp. Br J Dermatol 1977; 96:245-8.

8. Karen JK, Pomeranz MK. Skin changes and diseases in pregnancy. In: Wolff K, Goldsmith LA, Katz SI, Gilchrest BA, Paller AS, Leffell DJ, editors. Dermatology in General Medicine, 7th ed. New York: McGraw-Hill 2008:955-62.

9. Vazquez M, Maldonado $\mathrm{H}$, Benaman $\mathrm{C}$, et al. Melasma in men: A clinical and histologic study. Int J Dermatol 1988; 27:25-7.

10. Grimes PE. Melasma: Etiologic and therapeutic consideration. Arch Dermatol 1995; 131:1453-7.

11. Nguyen TH, Rooney JA. Trichloroacetic acid peels. Dermatol Ther 2000; 13:173-82.

12. Kumari R, Thappa DM. Comparative study of trichloroacetic acid versus glycolic acid chemical peels in the treatment of melasma. Indian Journal of dermatology 2010; 76:447.

13. Moy LS. Superficial chemical peels with alpha-hydroxy acid. In: Robinson JK, Andt KA, Wintroub BU, editors. Atlas of Cutaneous Surgery, 1st ed. Philadelphia: WB Saunders 1995; 345-51.

14. Kalla G, Garg A, Kachhawa D. Chemiçal peelingglycolic acid versus trichloroacetic acid in melasma. Indian J DermatolVenereolLeprol 2001; 67:82-4.

15. Dogra A, Gupta S, Gupta S. Comparative efficacy of $20 \%$ trichloroacetic acid and $50 \%$ glycolic acid peels in treatment of recalcitrant melasma. J Pak Assoc Derma 2006; 16:79-85. 